\title{
Leveraging Information Technology in Automating School Management and Student Activities by Successfully Integrating a Java- based School Management Application Software
}

\author{
Oluwole O. Oyetoke \\ Covenant University/Electrical and Information Engineering, Ota, 234, Nigeria \\ E-mail: oluwoleoyetoke@ gmail.com, oluwole.oyetoke@ @ovenantuniversity.edu.ng
}

\begin{abstract}
Considering the rapidly growing world population and increased enrolment in full course primary school education around the world, this paper elucidates key means of leveraging Information Technology for the effective and efficient management of the increasing pressure associated with schools' administrative functions and students' basic activities. Primarily narrowing down to the basic African education framework, this paper sheds more light on the methods which can be adopted for the development of such processes through improved Information Technology platforms. In doing this, the design and implementation of the Jasper School Management System (a school management solution developed by the author) will be used as a case study. Also, a brief highlight of the impact this Information Technology initiative will have on institutions where it is deployed. The Jasper School Management Software being referenced was built using Java Programming Language in conjunction with MySQL. It was produced to help improve management activities of schools especially in developing countries of the world, with 8 major school management modules incorporated into it. Also, the software is open-source i.e., open to adaptations, improvements and free to download.
\end{abstract}

Index Terms-School Management System, Java, Information Technology, MySQL, Education, Automation, Software Development.

\section{INTRODUCTION}

The world's population continues its upwards scaling with a birthrate of $1.89 \%$ per annum (over 132 million births) [1] and above $2 \%$ in some countries in the Middle East, Sub-Saharan Africa, South Asia, Southeast Asia and Latin America [2]. This growth, coupled with the United Nation's progress in its Millennium Development Goal as to achieve total enrolment of children around the world in full course of primary schooling has led to a high level of pressure being exerted on the management personnel of some of the already existing schools. This is even more evident as enrolment in primary school education in developing regions of the world recently hit an all time high of over $90 \%$ [3].

It can be agreed that the failure of most of the existing schools to leverage the high level of speed, accuracy and comfort offered by the integration of Information Technology in the school's management functions and student activity, enervates the work efficiency and foments a lot of stress on management personnel.

It is factual that Information Technology, while an important area of study, in its own right is having a major impact across all curricula area, providing instant access to a wide variety of data and also challenging assessment methods [4].

Manually computing report sheets and keeping track of many management functions (e.g. memos, mails, school calendar of activities) have always been there for centuries; however, desktop computer technology having being introduced, has revolutionized how schools, businesses and institutions operate. Also, the development of an extraordinary range of software, coupled with improved networking capabilities have exponentially increased the level of impact made by Information Technology. This enormous level of change brought about by new technologies has had a significant level of effect on the way people live, work and play worldwide, challenging the traditional process of teaching, learning and managing of schools.

This paper will explore the rudiments of implementing a school management system and also take a look at the benefits it offers. The School Management System that will be used as a case study is the Jasper School Management System (JSMS).

\section{LITERATURE STUDY ON INFORMATION TECHNOLOGY AND ITS BENEFITS TO SCHOOLS AND BUSINESSES}

\section{A. Definition}

Information technology is defined as the use of computers and software to manage information. In some industries, it can be referred to as Management Information Systems or Information Services [5]. 


\section{B. History}

The term Information Technology in its modern sense first appeared in a 1958 article published in the Harvard Business Review [6]. This was when some group of researchers tagged a then emerging technology with the name 'Information Technology'. The technology in question included:

1. The Application of Statistical and Mathematical methods to decision making

2. The Technique of Processing

3. The Simulation of higher order thinking through computer programs

\section{Benefits}

Information Technology today provides us with a variety of learning resources and immediacy of information, most of which are centered on:

1. Collaborative Learning

2. Automated School/Business Administration

3. Multimedia approach to learning

\section{Key School Management Activities}

Moving a step further to examining the school's management functions and student activities so as to highlight the best methods, architecture and framework to use in the implementation of the software application solution, it is evident that the following functions are conventional for personnel leading such roles (i.e. the Head Teacher, Teacher, Librarian, Accountant and studentship roles).

\section{A. Head Teacher}

1. Recruits new employees

2. Manages teachers and assigns classes/duties to them.

3. Has authorized access to students' performance report sheets, therefore, directly or indirectly having part duties in managing the creation, validity and integrity of this data.

4. Has authorized access to employee and student bio-data, thereby, directly or indirectly having part duties in managing the creation, validity and integrity of this data.

5. Decides/sets school's session details e.g., number of weeks per term, maximum test and examination scores.

6. Collaborates with the school's accountant to determine the fees to be paid by each class student.

7. Collaborates with the school's Librarian to determine book loan periods (in days, weeks or months)

8. Tracks employee/teacher performance

9. Communicates important information to employees.
In summary, the Head Teacher oversees and makes the final decision on all key management directives and initiatives.

\section{B. Class Teacher}

1. Registers new students

2. Registers class subject

3. Sets up assessments tests and examinations

4. Collate students report card for the various terms

5. Monitors student performance

6. Receives memos from the head teacher

7. Manages class attendance records

8. Teaches the class students

\section{School's Librarian}

1. Registers new books into the library

2. Lend out books to students and employees

3. Return nooks to the library

4. Take an inventory of books in the library

\section{School's Accountant}

1. Takes records of paid fees.

2. Takes records of all school's cash expenses.

3. Disburses employee salary \& takes records of such payments.

4. At all instances, be able to provide a statistical analysis of the school's current financial standing.

\section{E. Student}

1. Learn effectively in class

2. Take assessments

\section{Automation Methodology (CASE Study: JASPER SCHOOL MANAGEMENT SYSTEM)}

\section{A. Definition of Basic Terms}

\section{1) User Interface}

It can also be termed a visual way of interacting with a computer [7].

\section{2) Database}

A database is a separate application that stores a collection of data [8].

\section{3) Java Database Connectivity (JDBC)}

A JDBC driver enables Java applications to connect to a database in a particular Data Base Management System and allows you to manipulate that database using the JDBC Application Program Interface (API) [9].

\section{4) Relational Database Management System (RDBMS)}

Nowadays, we use RDBM Systems to store and manage huge volumes of data. This is called relational database because all the data is stored into different tables and relations are established using primary keys or other keys known as foreign keys [10]. 


\section{5) $M y S Q L$}

MySQL is a fast, easy-to-use RDBMS released under an open source license being used for many small and big businesses [9]. MySQL pronounced 'My sequel' is free to use and supports large database, up to 50 million rows or more in a table, with a theoretical limit of 8 million terabytes.

\section{B. System Workflow}

Looking into the methods and approach taken to achieve the goal of automating the activities of the principal management staff and student activities, we will have to first of all; examine the system and its user structure

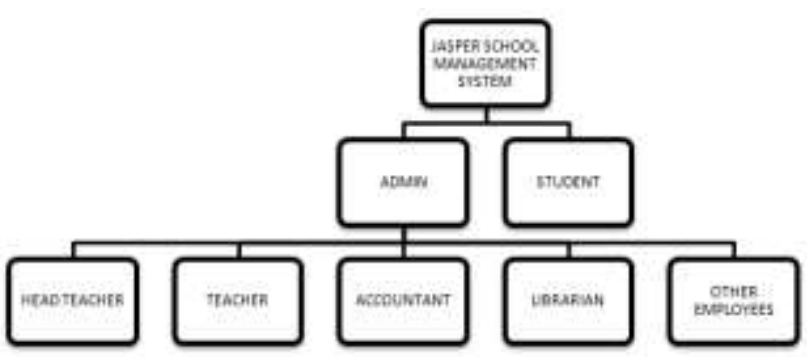

Fig.1. Overview of the System's User Structure

The software development was split into various modules, designed, built and tested separately at first and then in the end, all these modules integrated together as one unit. This is similar to Microsoft's Synchronize and Stabilize software development process model. By using the Java JDBC efficiently, the software is save important data and information in the database.

On first login, the default administrator is given the permission to input the school's name and the number of classes that will be run by the school (putting into consideration that the primary school education system of various countries differs). Immediately this is done, the system automatically creates the Persistence Layer, making available all the storage needs of the school. The Use Case Diagrams below describe other functions users of the systems can carry out (over 38 major school management functions and activities are made possible by the system).

The primary reason for the use case diagram is to model in a simple way, the interactions between a system's clients and its use cases. To show the kinds of interactions users have with a system without providing the details [11].

Fig. 2,3,4,5 and 6 below show the user interactivity of the Head Teacher, Teacher, Librarian, Accountant and Students on the system.

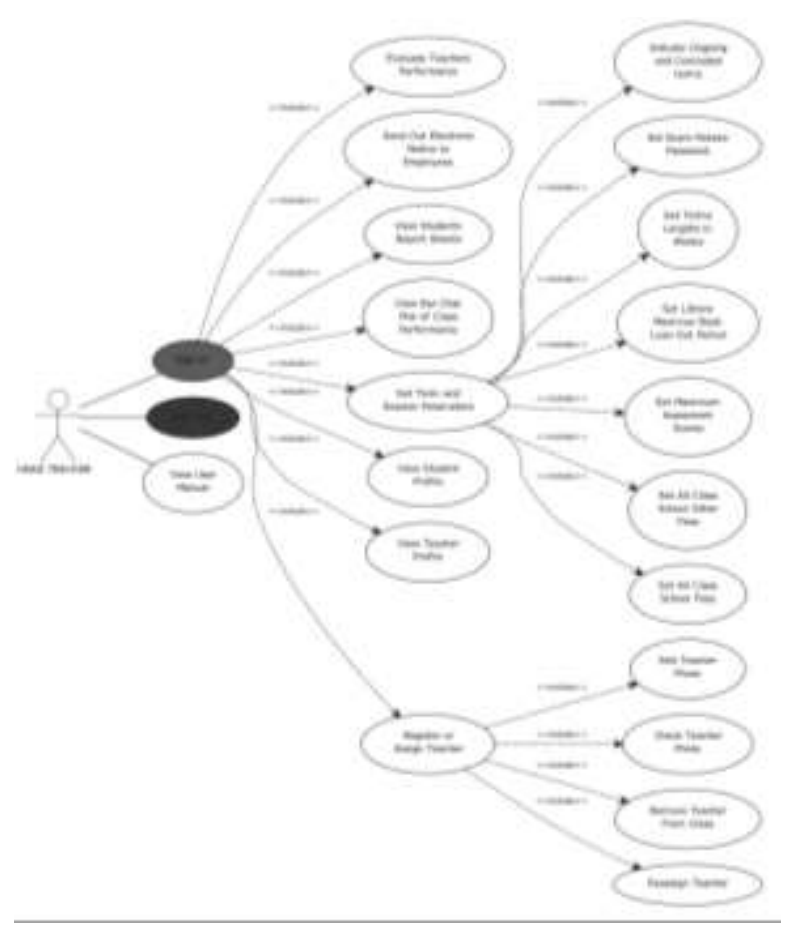

Fig.2. Head Teacher's Use Cases Use Case Diagram

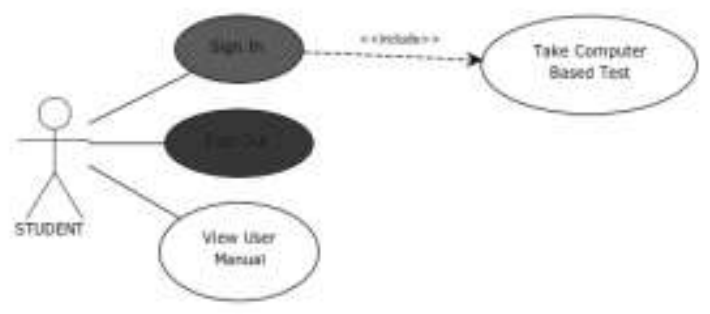

Fig.3. Student's Use Cases Use Case Diagram

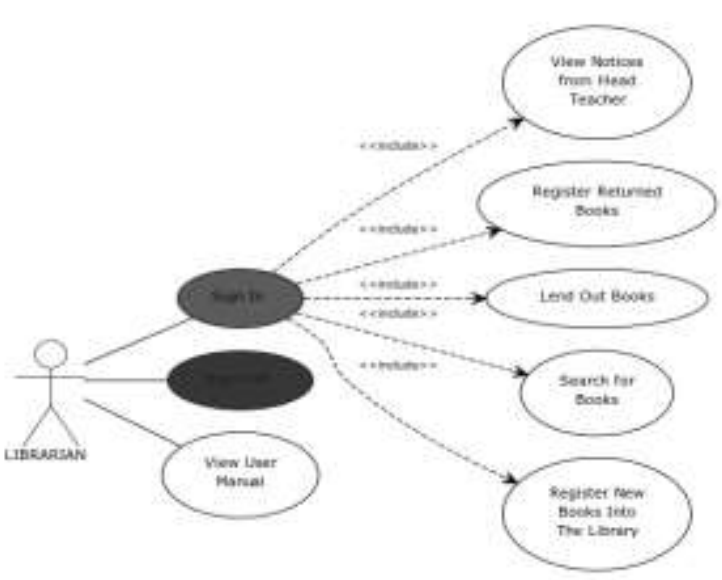

Fig.4. Librarian's Use Cases Use Case Diagram 


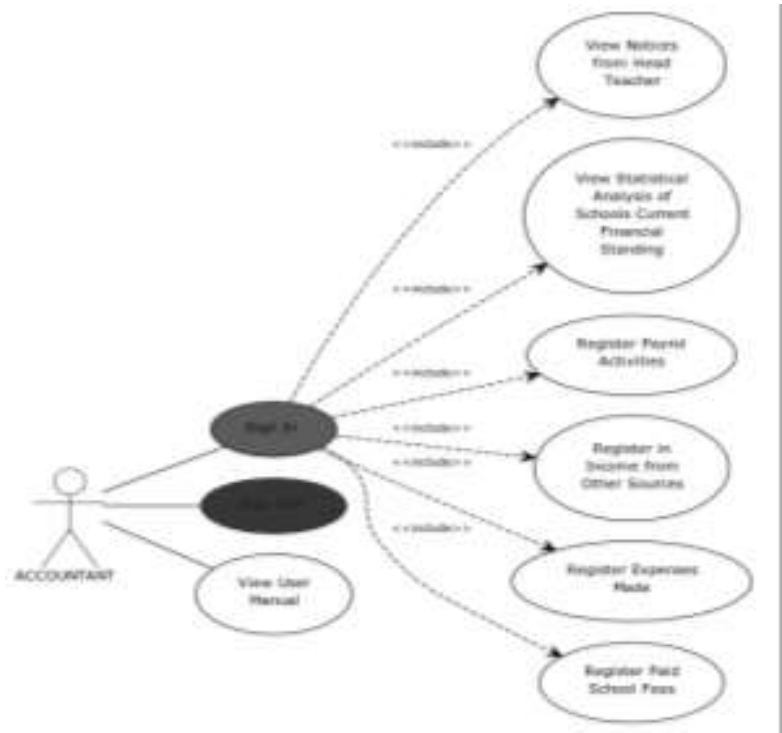

Fig.5. Accountant's Use Cases Use Case Diagram

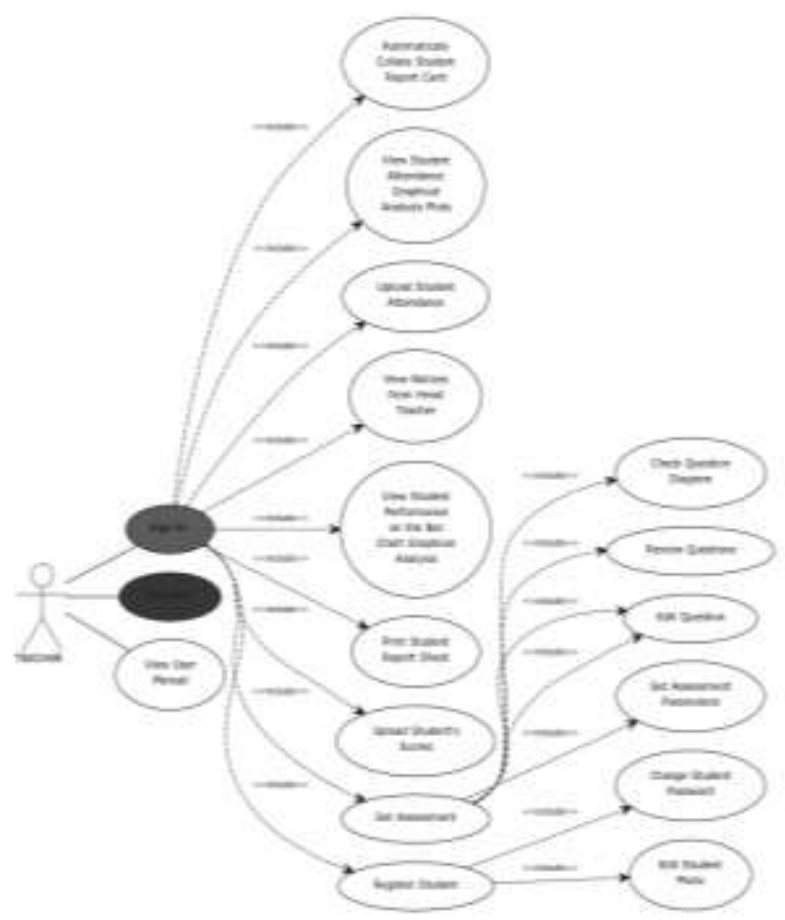

Fig.6. Teacher's Use Cases Use Case Diagram

\section{Plotting Mechanism}

The plotting of the class attendance data is made possible by the use of the Java Jmathplot Library. JMathTools is a collection of independent packages designed to fit common engineering/scientific computing needs. It contains:

- 'MathArray': simple linear algebra for double[ ] [ ] arrays

- 'JMath' Plot: interactive plot in 2D / 3D

- 'JMath' IO: binary input/output for double arrays [12].
The second plotting mechanism is another open source java library, the 'JOpenChart' library. It is a library and toolkit for rendering various kinds of charts. For this project, it helped in rendering the labeled bar charts.

\section{Statistical Analysis}

JSMS offers a wide range of statistical computational features, providing a means to instantly collate students' report sheets, analyze class average performance so as to understand and rate the class teacher's level of effectiveness. JSMS also furnishes the schools accountant with the tool to be able to view the school's financial standing and break down analysis at every instance of time

\section{Database Interactivity}

The desktop application continually interacts with the database using JDBC. With JDBC we can utilize the MySQL functionalities, using SQL queries and statements.

Table 1. Table Showing SQL Keywords and their Usage Syntax

\begin{tabular}{|c|c|c|}
\hline Keyword & Description & Usage Syntax \\
\hline SELECT & $\begin{array}{l}\text { Retrieves data from one } \\
\text { or more tables. }\end{array}$ & \multirow{2}{*}{$\begin{array}{l}\text { SELECT columnName FROM } \\
\text { tableName }\end{array}$} \\
\hline FROM & $\begin{array}{l}\text { Tables involved in the } \\
\text { query. Required in every } \\
\text { SELECT. }\end{array}$ & \\
\hline WHERE & $\begin{array}{l}\text { Criteria for selection that } \\
\text { determine the rows to be } \\
\text { retrieved, deleted or } \\
\text { updated. Optional in a } \\
\text { SQL query or a SQL } \\
\text { statement }\end{array}$ & $\begin{array}{l}\text { SELECT columnName } 1, \\
\text { columnName } 2, \ldots \text { FROM } \\
\text { tableName WHERE criteria }\end{array}$ \\
\hline $\begin{array}{l}\text { GROUP } \\
\text { BY }\end{array}$ & $\begin{array}{l}\text { Criteria for grouping } \\
\text { rows. Optional in a } \\
\text { SELECT query. }\end{array}$ & \\
\hline $\begin{array}{l}\text { ORDER } \\
\text { BY }\end{array}$ & $\begin{array}{l}\text { Criteria for ordering } \\
\text { rows. Optional in a } \\
\text { SELECT query. }\end{array}$ & $\begin{array}{l}1 . \quad \text { SELECT } \\
\text { columnName1, } \\
\text { columnName } 2, \ldots \text { FROM } \\
\text { tableName ORDER BY } \\
\text { column ASC } \\
\text { 2. SELECT columnName1, } \\
\text { columnName } 2, \ldots \text { FROM } \\
\text { tableName ORDER BY } \\
\text { column DESC }\end{array}$ \\
\hline INSERT & $\begin{array}{l}\text { Insert rows into a } \\
\text { specified table. }\end{array}$ & $\begin{array}{l}\text { INSERT INTO tableName } \\
\text { ( columnName } 1, \\
\text { columnName } 2, \ldots, \\
\text { columnNameN ) VALUES } \\
(\text { value } 1, \text { value } 2, \ldots, \text { valueN })\end{array}$ \\
\hline UPDATE & $\begin{array}{l}\text { Update rows in a } \\
\text { specified table. }\end{array}$ & $\begin{array}{l}\text { UPDATE tableName SET } \\
\text { columnName } 1=\text { value } 1, \\
\text { columnName } 2=\text { value } 2, \ldots, \\
\text { columnNameN }=\text { valueN } \\
\text { WHERE } \text { criteria }\end{array}$ \\
\hline DELETE & $\begin{array}{l}\text { Delete rows from a } \\
\text { specified table. }\end{array}$ & $\begin{array}{l}\text { DELETE FROM tableName } \\
\text { WHERE criteria }\end{array}$ \\
\hline
\end{tabular}

Each SQL keyword is processed by selected JDBC API methods. In general, to process any SQL statement with JDBC, the program follows these steps:

(1). Establish a connection

(2). Create a statement

(3). Execute the query

(4). Process the 'ResultSet' object

(5). Close the connection [13] 


\section{- Establishing Connections}

First, establish a connection with the data source (e.g. DBMS) you want to use, using a corresponding JDBC driver (e.g. SQL JDBC driver, if you are connecting to an SQL database). This connection is represented by a Connection object [13]. On first login, the user is prompted to enter the necessary details with which the JSMS can always access the database, to store, retrieve and modify the data therein, in a relational manner. These needed details are :

(1). The Database URL

(2). The Database Port Number

(3). The Database User Name

(4). The Database Password

\section{- Creating Statements}

A Statement is an interface that represents an SQL statement. You execute Statement objects, and they generate 'ResultSet' objects, which is a table of data representing a database result set. You need a Connection object to create a Statement object [13].

There are three different kinds of statements which are:

(1) Statement: Used to implement simple SQL statements with no parameters.

(2) 'PreparedStatement': (Extends Statement.) Used for pre-compiling SQL statements that might contain input parameters. See Using Prepared Statements for more information.

(3) 'CallableStatement': Used to execute stored procedures that may contain both input and output parameters.

\section{- $\quad$ Executing Querys}

To execute a query, call an execute method from Statement such as the following:

Execute: Returns true if the first object that the query returns is a 'ResultSet' object. Use this method if the query could return one or more 'ResultSet' objects. Retrieve the 'ResultSet' objects returned from the query by repeatedly calling 'Statement.getResultSet'.

(1) 'executeQuery': Returns one 'ResultSet' object.

(2) 'executeUpdate': Returns an integer representing the number of rows affected by the SQL statement. Use this method if you are using INSERT, DELETE, or UPDATE SQL statements.

\section{- Processing 'ResultSet' Objects}

You access the data in a 'ResultSet' object through a cursor. Note that this cursor is not a database cursor. This cursor is a pointer that points to one row of data in the 'ResultSet' object. Initially, the cursor is positioned before the first row. You call various methods defined in the 'ResultSet' object to move the cursor.

\section{- Closing Connections}

When you are finished using a Statement, call the method 'Statement.close' to immediately release the resources it is using. When you call this method, its 'ResultSet' objects are closed.

Code 1: Sample Code Illustrating Basic Java Database Interactivity

package tests;

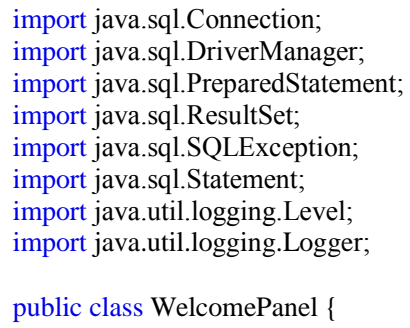

Connection testConnect; //Manages Connection Statement testStatement; //Query Statement

ResultSet testResult; //Manages Results

PreparedStatement testPrepare; //Manages Inserts and //Updates

String IPAddress;

String UserName;

String PassWord;

String PortNumber;

public static void main(String[] args) \{

WelcomePanel welcome = new WelcomePanel();

welcome.connect(); \}

public void connect() \{

IPAddress = "localhost";

PortNumber = "3306";

UserName = "root";

PassWord = "";

String criteria = "example";

//CONNECT TO DATABASE

try \{

Class.forName("com.mysq1.jdbc.Driver");

testConnect $=$ DriverManager.getConnection $(" j d b c:$ mysql://"

+ "'" + IPAddress + ":" + PortNumber + "'

+ "/databasename?autoReconnect=true", "'

+ "' + UserName + "', "' + PassWord + "');

testStatement $=$ testConnect.createStatement();

\} catch (Exception ex) \{

System.out.println("Unable to Connect " + ex); \}

//SELECT FROM DATABASE

try \{

String query $=$ "select $*$ from databasename where $"$

+ "columnName="' + criteria + "'";//* means to select //everything testResult $=$ testStatement.executeQuery(query);

int $\mathrm{c}=0$;

while (testResult.next()) \{

String myRequest $=$ testResult.getString("columnName"); \}

\} catch (SQLException ex) \{

System.out.println("Error in Getting Details From" +"Database " + ex); \}

\section{//INSERT INTO DATABASE}

try \{

\footnotetext{
String query = "insert into databasename" +" (ColumnName1,

+"ColumnName2)"

+ "value (?,?)";

testPrepare $=$ testConnect.prepareStatement(query);

testPrepare.setString(1, "Argument1");
} 
testPrepare.setString(2, "Argument2"); testPrepare.executeUpdate();

\} catch (SQLException ex) \{

System.out.println("Error in Inserting Into Table " + ex);

\section{//UPDATE DATABASE}

try \{

String query = "update databasename set columnName1 =?, "

+ "columnName2=? where columnNamex=?";

testPrepare $=$ testConnect.prepareStatement(query);

testPrepare.setString(1, "Argument1");

testPrepare.setString(2, "Argument2");

testPrepare.executeUpdate();

\section{\} catch (SQLException ex) \{}

System.out.println("Error in Updating Table " + ex); \}

\section{//DELETE FROM DATABASE}

try \{

String Query = "delete from databasename where "

+ "columnName="' + criteria + "'";

testStatement $=$ testConnect.createStatement();

testStatement.executeUpdate(Query);

\} catch (Exception ex) \{

System.out.println("Error in Deleting From Table " + ex); \}

//CLOSE CONNECTION

try \{

testConnect.close();

\} catch (SQLException ex) \{

System.out.println("Error in Closing Connection " + ex);

\}

\}

\section{Image Storage Mechanism}

To store images in the database, e.g. the profile pictures of employees the Java 'JFileChooser' helps in selecting the image file (ending with a .JPG, .PNG or .GIF). Thereafter, the file is collected into the file input stream, converted. and written into a byte array as bytes of data and this byes is inserted into the database.

To retrieve the image from the database, the bytes of data is queried and placed in a Byte Input Stream which is then read and reconstructed into the image.

Code 2: Excerpt of Code for Selecting and Converting Image Files to Bytes of Data

\section{private void}

uploadiagramButtonActionPerformed(java.awt.event.ActionEvent

evt) \{

JFileChooser fileChooser $=$ new JFileChooser();

FileNameExtensionFilter filter $=$ new FileNameExtensionFilter("JPG

\& GIF Images","jpg","png");

fileChooser.setFileFilter(filter);

fileChooser.setAcceptAllFileFilterUsed(false);

fileChooser.setFileSelectionMode(JFileChooser.FILES AND DIRECT

ORIES );

int result $=$ fileChooser .showOpenDialog $($ this $)$;

if (result== JFileChooser. CANCEL OPTION)

$$
\text { \} }
$$

else if(result==JFileChooser. $A P P R O V E$ OPTION) \{

java.io.File fileName $=$ fileChooser.getSelectedFile () ;

if $(($ fileName $==$ null $) \|($ fileName.getName () .equals $(" ")))\{$
JOptionPane.showMessageDialog(this, "Please Choose A Valid +"File", "FILE ERROR", JOptionPane.ERROR_MESSAGE);

else if(fileName.isFile ()$)\{$

String filepath $=$ fileName $\cdot$ getAbsolutePath();

try \{

File thefile $=$ new File(filepath)

FileInputStream $\mathrm{f}=$ new FileInputStream(thefile);

ByteArrayOutputStream BArray = new ByteArrayOutputStream(); try \{ byte[] buf = new byte[1024];

for(int readnum $=0$; $($ readnum $=$ f.read(buf) $) !=-1 ;)\{$

BArray.write(buf,0,readnum);

questionImage $=$ BArray.toByteArray () ;

\} catch (IOException ex) \{

JOptionPane.showMessageDialog(this, "Please Choose A Valid File " +ex, "FILE ERROR", JOptionPane.ERROR_MESSAGE);

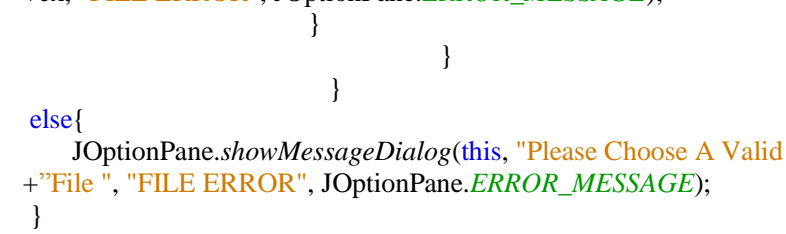

$\operatorname{try}\{$

String query1 = "select $*$ from tableName

parameter='argument'";

Result $=$ Statement.executeQuery(query1)

byte[] Diagram = new byte[1024];

while $($ Result.next ()$)\{$

Diagram = Result.getBytes ("Diagram");

if (Diagram $==$ null $)\{$

\}

displayImage.setIcon(null)

else \{

InputStream imageInput $=$ new

ByteArrayInputStream(Diagram);

BufferedImage Picture;

try \{

Picture = ImageIO.read (imageInput);

ImageIcon image $=$ new ImageIcon(Picture);

displayImage.setIcon(image); //Display Image in the text

\} catch (IOException ex) \{

Logger.getLogger(ExamPanel.class.getName()).log(Level.SEVERE, null, ex);

$$
\begin{gathered}
\}^{\}} \\
\text {\} catch (SQLException ex) }\{
\end{gathered}
$$

Logger.getLogger(ExamPanel.class.getName()).log(Level.SEVERE, null, ex); return;

\}

\section{GUI Creation}

Some Integrated Development Environments (IDE) provide GUI design tools with which you can specify a component's exact size and location in a visual manner by using the mouse while the IDE generates the GUI 
code itself. The creation of the JSMS's GUI was done with the Netbeans IDE drag and drop functionality. Focus was placed on the theme appeal and GUI attractiveness to the end user. Also, effort was made to create a simple and consistent outlook. This was aimed at giving the users, a level of familiarity with the system right from the first usage, so that they can learn the system's usage quickly and use it more productively.

\section{Database Creation}

A database schema is the skeleton structure that represents the logical view of the entire database. It defines how the data is organized and how the relations among them are associated. It formulates all the constraints that are to be applied on the data [14]. Table 3 below shows an excerpt of the database schema for JSMS.

Table 2. Table Showing an Excerpt of the Database Schema for JSMS

\begin{tabular}{|c|c|c|c|}
\hline \multicolumn{2}{|c|}{ question paper tabl } & \multicolumn{2}{|r|}{ expense_table } \\
\hline PK & Serial_Number & PK & Transaction_Code \\
\hline & $\begin{array}{l}\text { Question } \\
\text { Option A } \\
\text { Option B } \\
\text { Option C } \\
\text { Option D } \\
\text { Answer } \\
\text { Mark } \\
\text { Diagram } \\
\text { Diagram_Name } \\
\text { Term } \\
\text { Class } \\
\text { Enrolment_Key }\end{array}$ & & $\begin{array}{l}\text { Title } \\
\text { Amount } \\
\text { Description } \\
\text { Receipt } \\
\text { Date } \\
\text { User_Name } \\
\text { Paid_To } \\
\text { Term }\end{array}$ \\
\hline & notice_table & stu & t_details_table \\
\hline PK & Title & PK & User_Name \\
\hline & $\begin{array}{l}\text { Information } \\
\text { Postee } \\
\text { Position } \\
\text { Class } \\
\text { Time } \\
\text { Category }\end{array}$ & & $\begin{array}{l}\text { Serial Number } \\
\text { First_Name } \\
\text { Last_Name } \\
\text { Gender } \\
\text { Age } \\
\text { Class } \\
\text { Password } \\
\text { Parent_Number } \\
\text { Address } \\
\text { Comment } \\
\text { Picture }\end{array}$ \\
\hline
\end{tabular}

\section{IMPLEMENTATION}

Hardware and Software in Information Technology are mutually exclusive to each other. In a nutshell, Software functions on hardware. To successfully implement the JSMS application software in a school, various facilities have to be put in place, especially hardware facility. Although the implementation cost of the system in an environment with no prior hardware facilities might be high, its efficacy is immense in the long term. This section narrows down into the hardware, software and other necessary requirements for the full scale implementation of the JSMS in a school environment

\section{A. Hardware Requirements for Implementations}

\section{1) Switch}

A network switch (also called switching hub, bridging hub, officially MAC bridge) is a computer networking device that connects devices together on a computer network, by using packet switching to receive, process and forward data to the destination device [15].

\section{2) Server}

A server is a software program, or the computer on which that program runs, that provides a specific kind of service to client software running on the same computer or other computers on a network [16].

\section{3) Category 5 Cable (Cat 5)}

This is a twisted pair cable for carrying signals. This type of cable is used in structured cabling for computer networks such as Ethernet. The cable standard provides performance of up to $100 \mathrm{MHz}$ and is suitable for 10BASE-T, 100BASE-TX (Fast Ethernet), and 1000BASE-T (Gigabit Ethernet) [17].

4) A Computer or Series of Computers with Available Disk Space of Not Less Than 124MB and 512MB of RAM

\section{B. Software Requirements for Implementation}

1) Windows Operating System: Windows 8 (Desktop), Windows 7, Windows Vista SP2, Windows XP

\section{2) Java runtime Environment (JRE 7) and Above}

\section{Usage in A Networked Environment}

The Jasper School Management System is designed to be utilized in a networked environment, affording the school's employees and multiple students the opportunity to use the system simultaneously, with only one computer as the server (connected to as many more computers). With this, administrative, management and various Computer-Based Testing activities can be performed with ease. Some steps, procedures and considerations need to be put in place before this functionality can be fully utilized. Most of these considerations are centered on creating a wired local area network with little or no firewall restrictions.

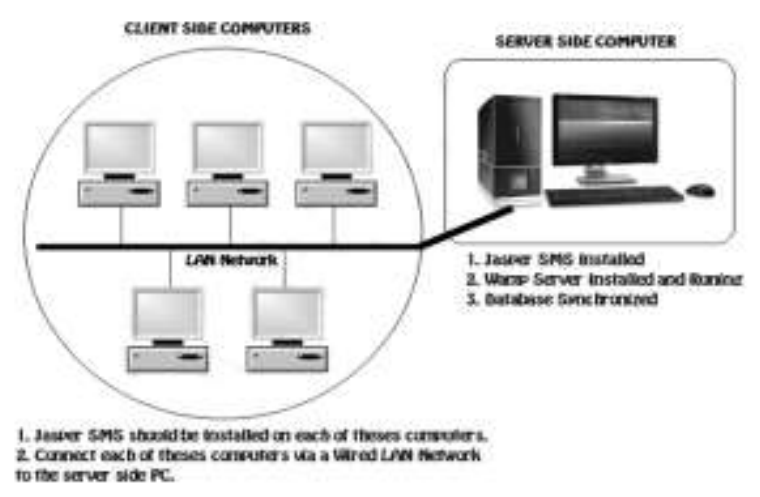

Fig.7. Overview of JSMS Usage in a Networked Environment. 


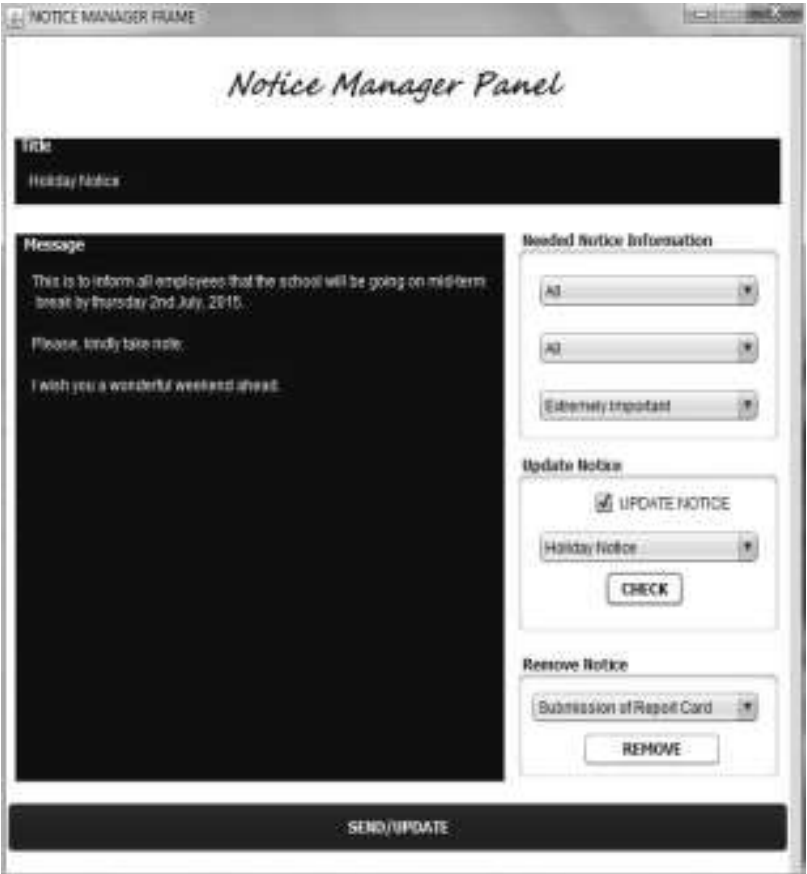

Fig.8. Screenshot of the School's Communication/Notice Manager Panel.

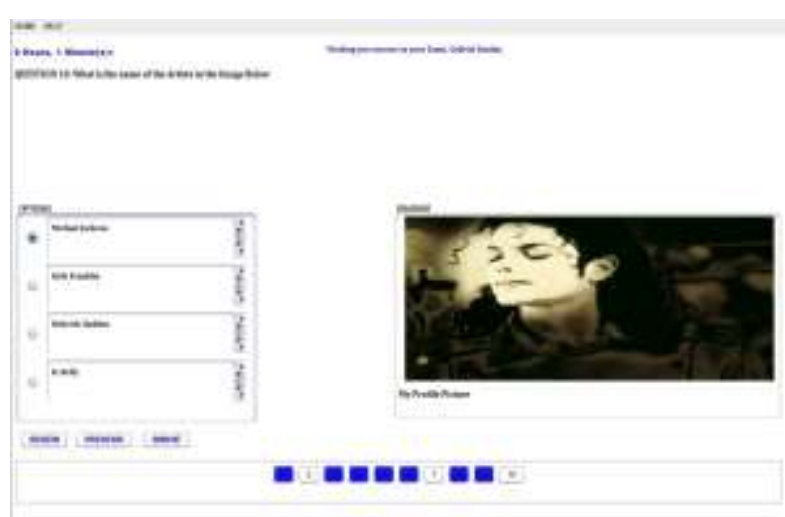

Fig.9. Screenshot of an Ongoing Compute-Based Test on JSMS

After the network has been created and the database is synchronized on the server, the Jasper School Management software is installed on the various other computers which will be connecting to the server for resources. These other computers do not need database synchronization. This is because they will be pulling resources from the main computer which is the server.

\section{Screen-Shots of the Fully Operational System}

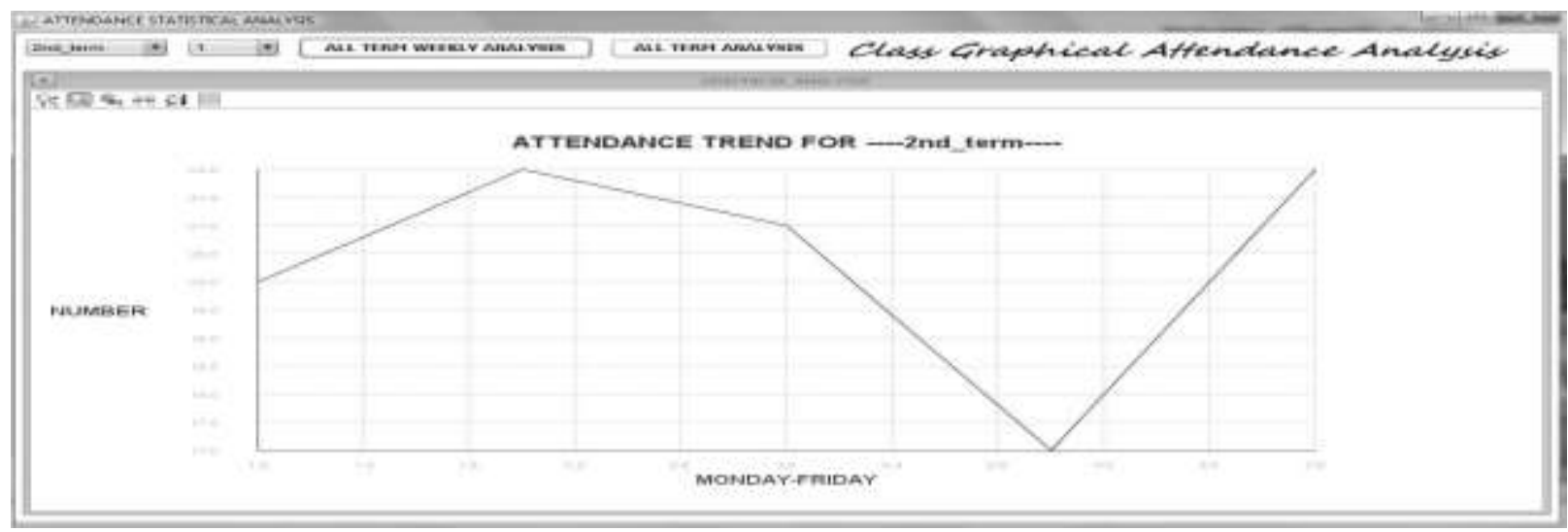

Fig.10. Screenshot of the Class Attendance Data Plotter Frame Accessible To The Class Teacher

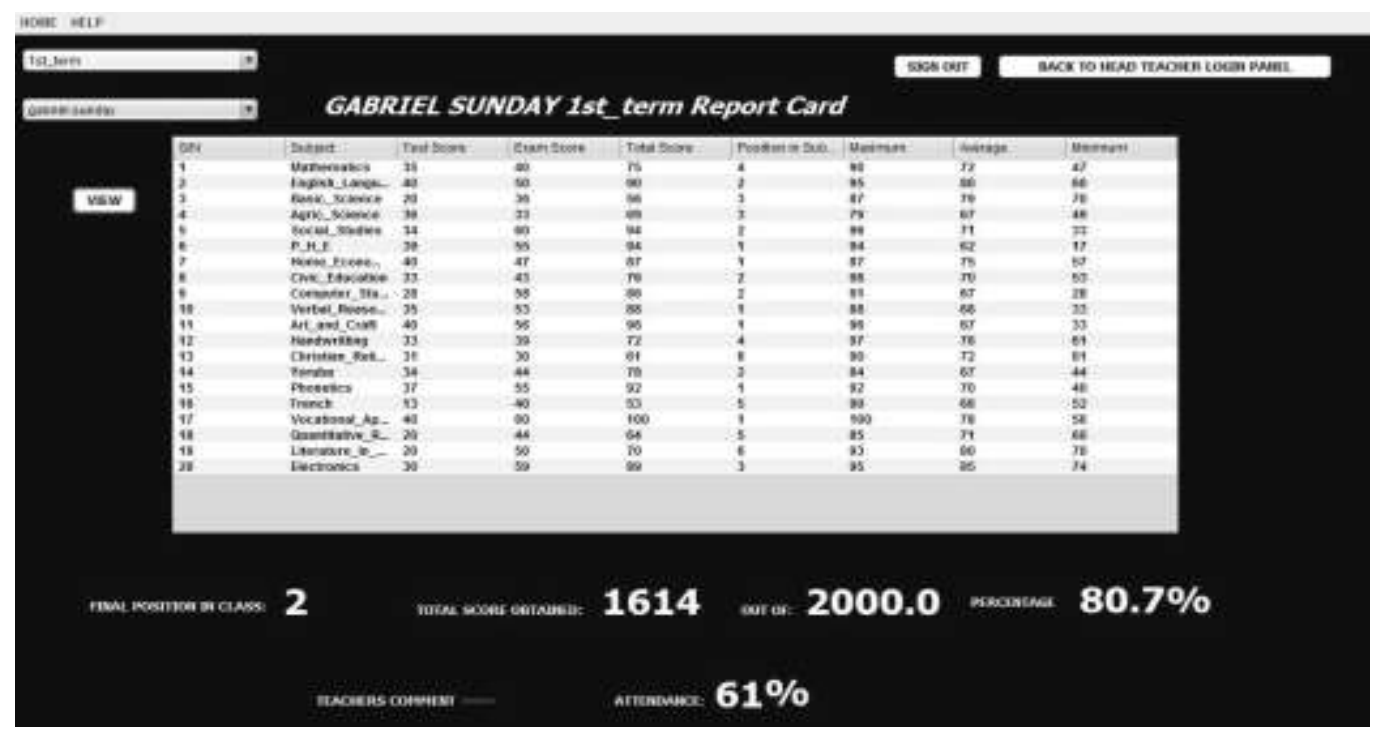

Fig.11. Screenshot of the Automatically Generated Student Result Sheet Accessible to the Head Teacher 


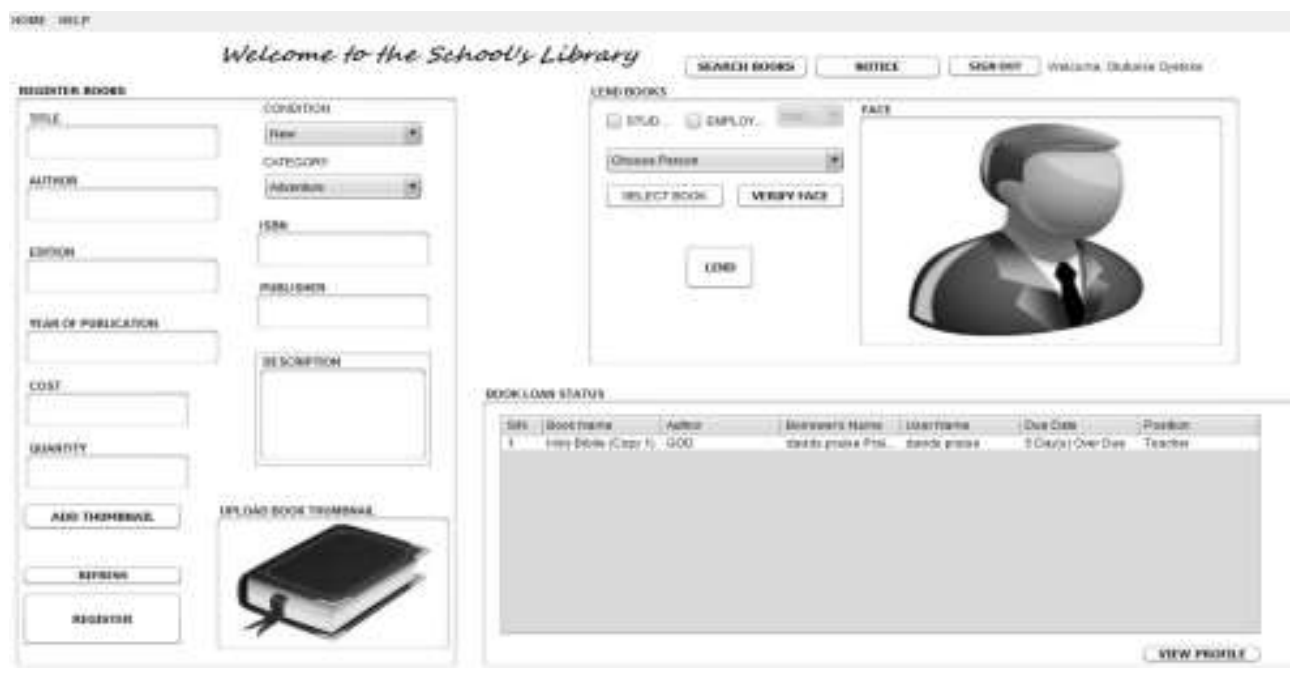

Fig.12. Screenshot of the User Interface of the Librarian's Library Management Module Incorporated into the JSMS

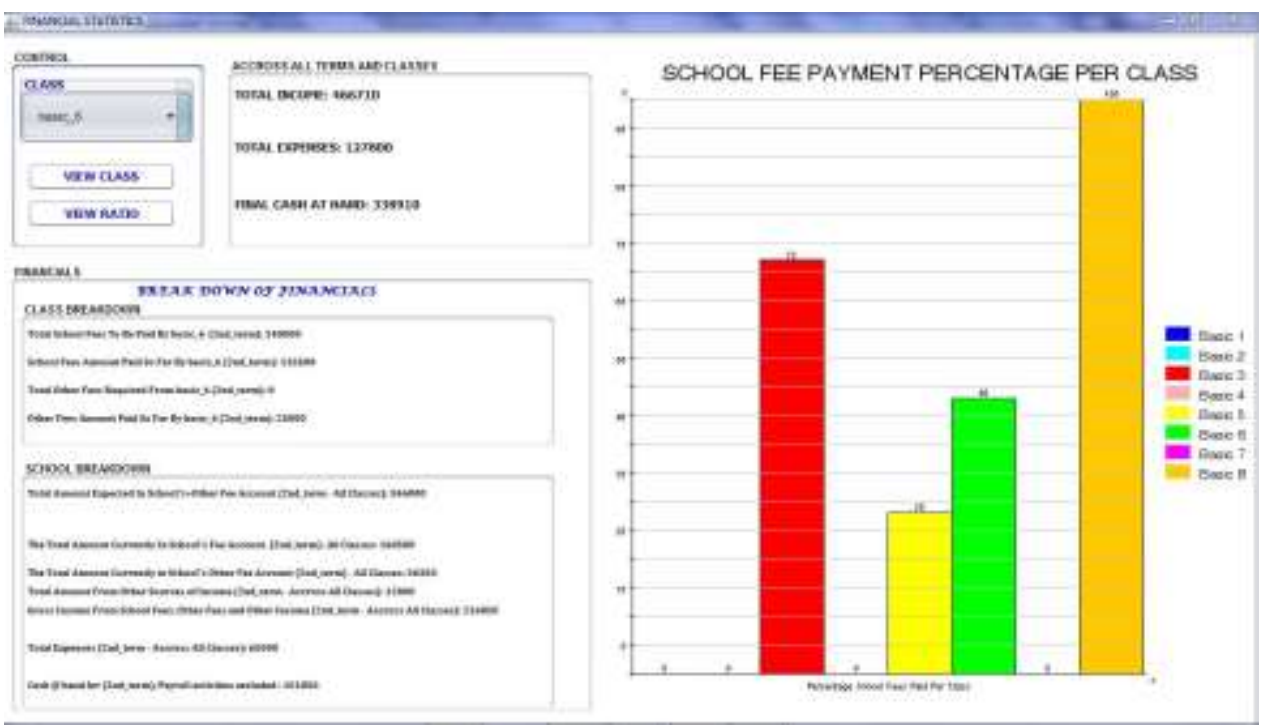

Fig.13. Screenshot of the User Interface of the Accountant's query of the School's Current Financial Breakdown via the Finance Management Module, incorporated in the JSMS

\section{COST OF FULL IMPLEMENTATION}

Implementing Information Technology solutions can at first be costly. This is because a lot of resources have to be put into purchasing hardware resource. The table 3 below highlights a breakdown of the cost implications of implementing JSMS in an averagely populated school.

\section{IMPACT ANALYSIS}

The impact of fully implementing Information Technology solutions are contextual i.e., they differ based on the area of application. In some areas, IT offers majorly security, in some, speed and accuracy, and various other benefits as the case may be.

Explicitly examining the benefits of this JSMS IT solution, we can anchor its impact on four major areas which are highlighted below:
Table 3. Table Showing the Cost Analysis for the Implementation of JSMS in An Institution ${ }^{1}$

\begin{tabular}{|c|c|c|c|}
\hline EQUIPMENT & QUANTITY & DESCRIPTION & $\operatorname{COST}(\$)^{2}$ \\
\hline Router $^{3}$ & 1 & $\begin{array}{l}\text { Integrated services } \\
\text { router with }\end{array}$ & $\$ 365$ \\
\hline Switches & Varies & $\begin{array}{l}\text { Cisco Catalyst } \\
\text { Switch }\end{array}$ & $\$ 365$ \\
\hline $\begin{array}{l}\text { CAT5e } \\
\text { Cables }\end{array}$ & Varies & & $\begin{array}{c}\$ 0.5 \text { per } \\
\text { metre }\end{array}$ \\
\hline 1 Server & 1 & $\begin{array}{c}\text { Octa-Core Intel } \\
\text { Xeon X3 CPU, 2GB } \\
\text { of DDR3 RAM, } \\
\text { 250GB SATA drive. } \\
\text { maximum of 16GB } \\
\text { RAM } \\
\end{array}$ & $\$ 700$ \\
\hline $\begin{array}{l}\text { Back Up } \\
\text { Power } \\
\text { (Inverter + } \\
\text { Batteries) for } \\
\text { Server }\end{array}$ & 1 & $\begin{array}{c}2.5 \mathrm{kVA} / 48 \mathrm{~V} \\
\text { Inverter }+ \\
\text { Deep Cycle } \\
\text { Batteries }+ \\
\text { Installation } \\
\end{array}$ & $\$ 1759$ \\
\hline $\begin{array}{c}\text { Software } \\
\text { (JSMS) }\end{array}$ & & Installation & $\$ 0$ (Free) \\
\hline Total & & & $>=\$ 3189$ \\
\hline
\end{tabular}




\section{1) Speed}

- The teacher can now compute students' attendance aggregate in less than 5 seconds.

- Students' end of term report sheets can now be computed at the click of a button

- Students' assessments result is now automatically made available to the teacher immediately student finishes the assessment.

- The School's Accountant can get the school's financial standing in an instance, without having to manually gather records together for computation.

- The computerized library management module incorporated into it allows the Librarian speed up his/her bookkeeping tasks

In summary, all computational tasks in the management role have been completely automated and sped up.

1- Analysis Excludes the purchase of computer systems for the school or the setting up of a functional computer lab.

2- Prices are given on 'as is' basis and are stated as of July, 2015.

3- You will only need a router in the case where the school has a large network, therefore springing up the need to link two or more network domains together.

\section{2) Accuracy/Precision}

- The Librarian now can accurately track books in and out of the library.

- Students' assessment grading will be void of human errors due to the computer based testing module incorporated.

- Students' result sheets can now be collated with little or no error recordings.

- The communication module offers the option of selecting the intended recipient of a particular memo, thereby ensuring precise communication as well as information integrity (the intended recipient gets the message in his/her portal, unadulterated).

- Considering the fact that any endeavour that deals with figures requires accurate calculations and results, the JSMS will definitely enhance the accuracy and precision with which the schools financial standing is calculated.

\section{3) Efficiency}

- The School's Head Teacher can now efficiently circulate memos to selected employees in an electronic manner.

- Examinations can be disbursed to a large group of students in batched, efficiently via the CBT system

- Because the JSMS holistically automates a large percentage of the schools employees' activities/task, it generally improves their work efficiency
- Considering the fact that financial and other calculations are now being achieved in a more accurate manner, this engenders efficiency on the path of the accountant

- Inefficiency earmarked by errors made during the scoring of students assessment tests will have been reduced to the barest minimum

\section{4) Improved Creativity}

- Time saved due to the level of automation introduced by this innovative approach can now be re invested by employees into other functions and creative endeavours.

- The automated processes will tamper stress on the employees, thereby stimulating their creative abilities.

\section{OPEN ACCESS INFORMATION}

To get access to this software, user manual, and source code for use and adaptation purposes, visit https://schoolmgt.oluwoleoyetoke.com or http://sourceforge.net/projects/primaryschoolmanagement system/. This will offer intending users the opportunity to lay hands on quality products, as it agreed that open source software get closest to what users want because those users can have a hand in making it so.

\section{CONCLUSION}

Information Technology has today provided society with the channel through which iterative management activities of schools, businesses and organizations can be easily automated, consequently leading to increased level of efficiency, effectiveness, accuracy and possibly creativity. Leveraging Application Software development and the auspicious reach of networked ICT devices can indeed make the tasking job of managing big schools/organizations easier, lighter and most of all, enjoyable.

It is imperative to understand that process improvement is a key objective of Information Technology, allowing users to holistically review their business activities and execute needed task quickly. It can be gleaned from this paper that the integration of the Java software application into the school's processes will in summary, make employees achieve a lot more, more accurately and in far lesser time, thereby giving the employees ample opportunity to put their minds into judicious use i.e., other pertinent creative endeavours.

It can therefore be concluded, that, although the initial Information Technology implementation costs can be substantial, the resulting long-term impact are usually worth the investment. It allows companies reduce the time spent on iterative transaction while helping them gain and maintain competitive advantage, positioning them financially to grow locally and then internationally. 


\section{ACKNOWLEDGMENT}

I wish to acknowledge Jasper and Chrysolite Nursery and Primary School, Lagos, Nigeria, for giving me a one (1) month teaching opportunity which gave me insight on the current Nigerian Primary School Education structure. With this insight, I was able to design the various modules of the JSMS.

\section{REFERENCES}

[1] World Fact Book, www.cia.gov/library/publications/theworld-factbook/fields/2002.html , retrieved $10^{\text {th }} \mathrm{July}$, 2015

[2] Population Growth, http://enm.wikipedia.org/wiki/Population_growth, retrieved 12th July, 2015.

[3] MDG 2 infographics, http://un.org/milleniumgoals/education.shtml, retrieved 12th July, 2015.

[4] Importance of Information Technology in Educatuon, http://wikieducator.org/Need_and_Importance_of_Inform ation_Technology_in_Education , retrieved 13th July, 2015

[5] History of Information Technology, http://it.tmcnet.com/topics/it/articles/164702-historyinformation-technology.htm, retrieved 12th July, 2015.

[6] Leavit, Harold J. Whisler Thomas (1958), "Management In The 1980s", Harvard Business Review

[7] Graphical User Interface, http://google.com. Retrieved 17th May, 2015.

[8] Tutorials Point, 'MySQL Tutorial', Page 13.

[9] Paul and Harvey Deitel, 'Java How to Programme', 9th Edition, Page 1172, Patience Hall, 2012

[10] Tutorials Point, 'MySQL Tutorial', Page 13.

[11] Paul and Harvey Deitel, 'Java How to Programme', 9th Edition, Page 475, Patience Hall, 2012

[12] Jmath Plot, http://jmathtools.berlios.de/doku.php, retrieved; 17th January, 2014.

[13] Processing SQL Statements, http://docs.oracle.com/javase/tutorial/jdbc/basics/processi ngsqlstatements.html, retrieved 14th July, 2015.

[14] DBMS Data Schemas, http://www.tutorialspoint.com/dbms/dbms_data_schemas. htm , retrieved 14th July, 2015.

[15] Network

Switch, https://en.wikipedia.org/wiki/Network_switch, retrieved 14th July, 2015.

[16] Server, www.linfo.org/server.html, retrieved 14th July, 2015.

[17] Category 4 Cable https://en.wikipedia.org/wiki/Category_5_cable, retrieved 14th July, 2015.

\section{Author's Profile}

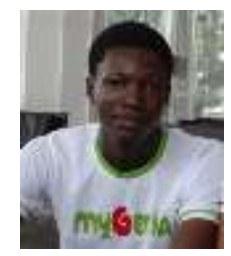

Oluwole O. Oyetoke is a citizen of Nigeria, West Africa. He is a graduate of information and communication engineering (Year 2014) at the Department of Electrical and Information Engineering, Covenant University, Ota. Nigeria.

In research, he has been focusing on the application and utilization of Information Technology in the improvement and development of the quality of delivery of education across African Institutions. Designing and Developing a Simulation package for data communication, aimed at contributing to Simulation Based Engineering Learning in third world universities lacking the financial manpower to purchase vital Communication Engineering hardware. His current interest is in Mastering in Embedded Systems Engineering.

Mr. Oyetoke is a member of IEEE (Institute of Electrical and Electronics Engineers) and also a member of NSE (Nigerian Society of Engineers). He has bagged various awards, including the Procter and Gamble 'Power of You' award, an award for being one of the most strategic employees in the first quarter of the 2014/2015 Fiscal Year in Nigeria.

How to cite this paper: Oluwole O. Oyetoke,"Leveraging Information Technology in Automating School Management and Student Activities by Successfully Integrating a Java- based School Management Application Software", International Journal of Information Technology and Computer Science(IJITCS), Vol.8, No.1, pp.22-32, 2016. DOI: 10.5815/ijitcs.2016.01.03 